\title{
Coupling structure for a high Q corrugated cavity as a microwave undulator
}

\author{
Liang Zhang, Wenlong He, Craig R. Donaldson, Jim Clarke, Kevin Ronald, Alan D. R. Phelps and \\ Adrian W. Cross
}

\begin{abstract}
Microwave undulators can achieve smaller wiggler periods when compared with permanent magnet undulators. This work follows on the design of a microwave undulator composed of a corrugated waveguide operating at $36 \mathrm{GHz}$. Compatible end caps and coupling structures for the waveguide to form a complete cavity were studied and are presented in this paper. Their design is scalable for different operating frequencies and periods of the corrugated waveguide. A set of empirical equations, that allows the geometry to be determined analytically, were found from the post-processing of the FDTD simulation.
\end{abstract}

Index Terms - microwave undulator, free electron laser, corrugated waveguide, coupler.

\section{INTRODUCTION}

$\mathrm{T}$ he undulator is one of the most important components in a free electron laser (FEL) [1, 2]. Short-wavelength radiation is generated when a relativistic electron passes through a periodic transverse magnetic field generated by an undulator [2]. The periodic transverse magnetic field can also be generated by an electromagnetic wave with the proper operating mode, which is the principle of a microwave undulator [3, 4].

Compared with an undulator composed of permanent magnets [5] or superconducting coils [6], the microwave undulator is advantageous due to its ability to achieve a short undulator period through operating at high frequency. Its polarization and the equivalent magnetic field strength can also be easily controllable by varying the polarization and power of the driving microwave source [7].

The microwave undulator can either operate in the standing wave mode by using a cavity structure or the backward traveling wave mode by using a waveguide structure [8-11]. A microwave undulator using a cavity composed of a corrugated waveguide operating at $\mathrm{X}$-band has been designed and tested in SLAC. It achieved an equivalent magnetic field $B_{u}$ of $0.65 \mathrm{~T}$ and an undulator period of $13.9 \mathrm{~mm}$, when it was driven by a $50 \mathrm{MW}$ klystron at $11.424 \mathrm{GHz}$ [11]. Using the same concept, a microwave undulator operating at $36 \mathrm{GHz}$ was proposed for the CompactLight and UK XFEL [12]. A microwave undulator

L. Zhang (liang.zhang@strath.ac.uk), C. R. Donaldson (craig.donaldson @ strath.ac.uk), K. Ronald (k.ronald@strath.ac.uk), A. W. Cross (a.w.cross@strath.ac.uk) and Alan D. R. Phelps (a.d.r.phelps@strath.ac.uk) are with Department of Physics, SUPA, University of Strathclyde, Glasgow, G4 0NG, Scotland, UK. L. Zhang and A. W. Cross are also with the Cockcroft Institute, Warrington Bank Quay. UK. J. Clarke (jim.clarke@stfc.ac.uk) is with the Cockcroft Institute, Sci-Tech Daresbury, and the ASTeC, STFC Daresbury with $1.75 \mathrm{~mm}$ period operating at $91.392 \mathrm{GHz}$ was designed and presented in publications [13,14].

To achieve a high $B_{u}$ comparable to the state-of-the-art permanent magnet undulator [15], the microwave undulator is required to operate with power at the tens of MWs level. The Ohmic loss therefore becomes an important issue. A smaller Ohmic loss not only reduces the thermal load, relieving the cooling requirement for such high power applications, but also increases the quality factor $\mathrm{Q}$. Since $B_{u}$ is proportional to $\mathrm{Q}^{1 / 2}$ when the input power and operating frequency are fixed, reducing the Ohmic loss will result in higher $B_{u}$ [12].

In the cavity-type microwave undulator, the Ohmic loss includes the loss from the waveguide wall and the caps at both ends. The $\mathrm{Q}$ factor is also determined by the coupling aperture. Once the operating frequency and mode are determined, the geometry of the waveguide, and thus its Ohmic loss, will be almost fixed leaving the geometry of the end cap an important issue. In papers [11, 13], a high Q cavity has been achieved using tapered waveguides and circular caps at both ends. The coupling apertures were placed at the positions of small field strength to minimize the impact on the eigenfrequency and the field pattern of the operating mode. The measurement results were excellent, however no details were presented. While in paper [12], only the properties of the corrugated waveguide were studied, and the coupling structure was not investigated. Although it is possible to get an acceptable design by carrying out a large number of parameter scans, it should be noted that the microwave undulator is usually long, with a scale of meters. The simulation time is long and requires large computing resources.

In this paper, the structure of the end caps and the positions of the coupling apertures were studied to maximize the $\mathrm{Q}$ factor and the design is presented in detail. The design methodology has the following advantages: (1) a set of empirical equations were found to quickly determine the parameter range of the geometry. (2) The design is scalable as the equations were normalized to the operating frequency. (3) The end caps and the coupling apertures will affect the properties of the microwave undulator cavity since it is part of the cavity. In this design, its

Laboratory, Sci-Tech Daresbury, Keckwick Lane, Daresbury, Cheshire, WA4 4AD, United Kingdom. W. He (wenlong.he@ szu.edu.cn) is with College of Electronic Science and Technology, Shenzhen University, Shenzhen, 518060, China. This work is supported by the European Commission Horizon 2020 Project "CompactLight" (777431-XLS). The support of the STFC UK (Cockcroft Institute Core Grant R160525-1) is gratefully acknowledged. 
impact was minimized. It can be designed at short undulator length and verified at longer undulator length to save the simulation time. (4) the design follows a natural approach and the same process can be used for the microwave undulator operating at other modes, for example, the higher order $\mathrm{HE}_{12}$ mode.

The paper is organized as follows. Section II summarises the properties of a cavity composed of the corrugated waveguide. Section III describes the empirical equations to determine the dimensions of the end caps. Section IV presents the simulation result of a corrugated cavity for the microwave undulator.

\section{THE PROPERTIES OF THE CORRUGATED WAVEGUIDE}

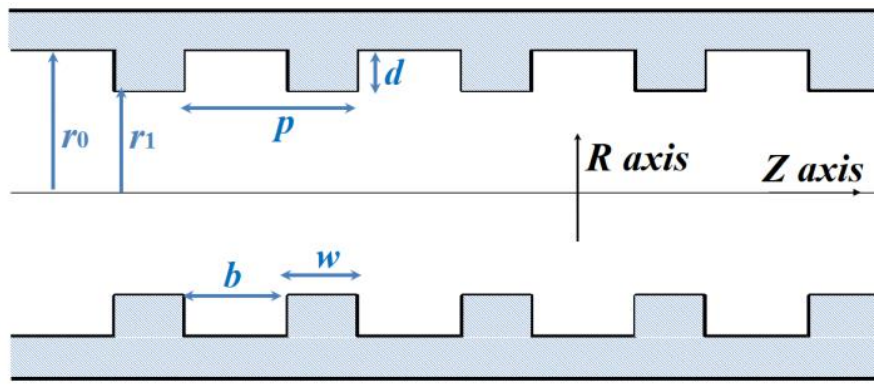

Fig. 1. Schematic drawing of the corrugated waveguide.

Corrugated waveguides, shown as Fig. 1, have been used in applications such as feed horns and transmission lines due to its low loss and wide bandwidth [16, 17]. The eigenmodes in the corrugated waveguide are the HE/EH modes. The dispersion relation of the corrugated waveguide can be accurately solved using the mode-matching method [18-20], in which the hybrid TE and TM modes are used. However it is not convenient to excite the hybrid TE and TM fields with precise amplitudes and phases at the same time in finite-difference time-domain (FDTD) simulations. Also, the content of the hybrid modes strongly depends on the geometry and it is difficult to provide a universal design. The dispersion relation can also be derived from the surface-impedance approach which has been derived in the papers $[10,12,16,21]$, when its dimensions satisfy the balanced hybrid condition and a large radius $r_{1}$ is used to satisfy $2 \pi r_{1} / \lambda_{0} \gg 1$. This enables the linearly polarized $\mathrm{HE}_{1 \mathrm{~m}}$ mode inside the corrugated waveguide to be simplified as follows:

$$
\left\{\begin{array}{l}
E_{z}=a_{m} J_{m}(K r) \cos (m \phi) \\
E_{r}=-j a_{m} \frac{k}{K} J_{m-1}(K r) \cos (m \phi) \\
E_{\phi}=j a_{m} \frac{k}{K} J_{m-1}(K r) \sin (m \phi)
\end{array}\right.
$$

where $\lambda_{0}$ is the wavelength in free space, and $K$ is the transverse wavenumber. The dispersion curve can be solved from the simplified equation $J_{0}\left(K r_{1}\right)=0$. The first two modes $\mathrm{HE}_{11}$ and $\mathrm{HE}_{12}$ have the solutions of $K=2.4 / r_{1}$ and $5.5 / r_{1}$, corresponding to $m=1$ and $m=2$ respectively.

A cavity could be formed simply by closing both ends of the corrugated waveguide. However, in this case, the coupling apertures would have to be placed on the end wall. Because the operating mode had the maximum magnetic field on the end caps, the coupling aperture would have a strong influence on the eigenfrequency and distort the field pattern of the operating mode.
To reduce the loss at the end walls, it is essential to have small electric and magnetic fields at the boundary, as is the case for the side wall of the corrugated waveguide. The problem can, therefore, be converted to find a perfect electrical conductor (PEC) boundary where the electric and magnetic field strengths are small. This can be achieved by radiating the $\mathrm{HE}_{1 \mathrm{~m}}$ mode into free space, then to determine the PEC boundary from the nearfield radiation pattern.

In this study, the FDTD method was used to simulate the near-field radiation pattern. To obtain accurate results the field at the end of the corrugated waveguide should match with the field pattern of the operating mode from the eigenmode solver. This means that it is important to extract the near-field radiation pattern at the right time. It is also important that the correct mode is excited in the waveguide. Many commercial FDTD solvers do not offer an easy way to excite the required $\mathrm{HE}_{1 \mathrm{~m}}$ mode through a circular waveguide port, for example, CST Microwave Studio, only excites TE, TM modes or their combinations at the port.

The open-sourced electromagnetic field solver OpenEMS was used in these simulations [22]. OpenEMS solves the 3D electromagnetic problems using the FDTD method. The core of OpenEMS utilizes $\mathrm{C}++$ and enables multithreading calculations, therefore allowing a high-speed simulation. The biggest advantage of OpenEMS is that it provides a MATLAB interface and the whole simulation process can be controlled through a simple MATLAB script. This allows quick implementation of the simulation and data post-processing of the results, which benefits from the powerful in-built MATLAB functions. OpenEMS also allows flexible control of the simulation process including defining a custom excitation signal and field pattern at user-defined positions. The field of the HE modes can be excited using Eq. 1.

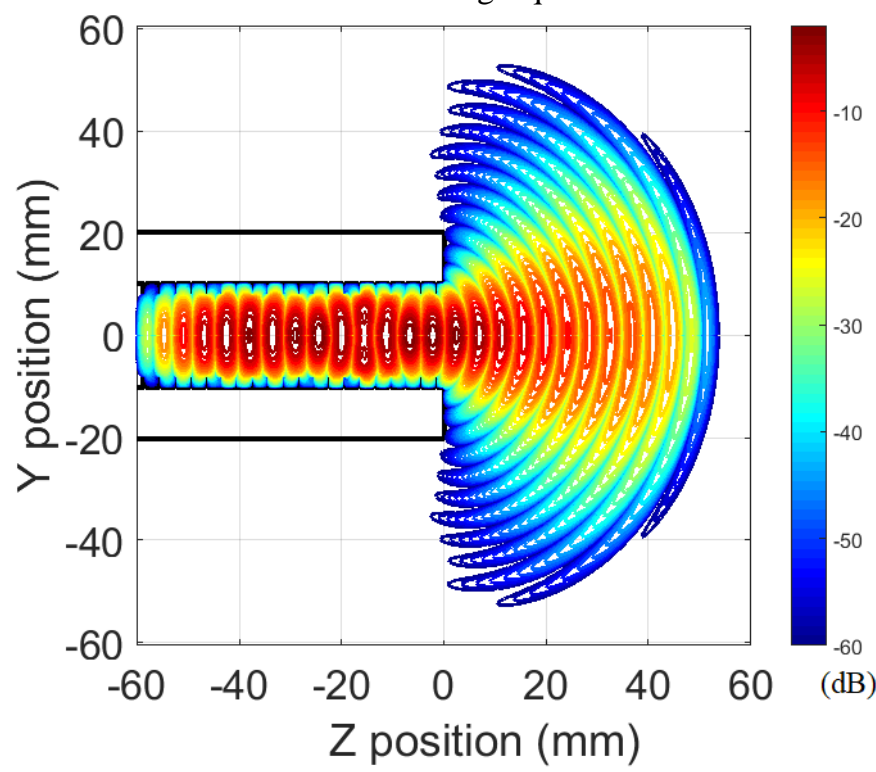

Fig. 2. Near-field radiation pattern of the corrugated waveguide at $X=0$.

The simulated structure was a few periods of the corrugated waveguide. The $\mathrm{HE}_{11}$ mode was excited at the middle of the waveguide. The left-hand side of the waveguide was set as a waveguide port to reduce the possible reflections. At the righthand side, it was set as radiating the electromagnetic wave into free space to explore the zero-field boundary. The simulation 
region in the radial direction was set to be 5 times larger than the radiation aperture so that there would be no reflection from the simulation boundary. A fine mesh grid was used to allow adequate spatial resolution for post-processing of the simulation result. The electric fields of each time step at the $X=0$, shown in Fig. 2, where the $\mathrm{Y}=0$ and $\mathrm{Z}=0$ planes were exported for further analysis.

\section{EMPIRICAL EQUATIONS TO DETERMINE THE DIMENSIONS OF THE COUPLING STRUCTURE}

The $-40 \mathrm{~dB}$ contour line was used to determine the boundary of the end caps and coupling structures. From the near-field radiation pattern shown in Fig. 2, the 'small-field' boundary could be defined as in Fig. 3, which was similar to the one used in papers $[11,23]$. The geometry was governed by a few parameters marked in Fig. 3. The taper part allows a tapering field strength and it helps to avoid oscillation caused by the relativistic electron bunch experiencing an abrupt field. It also provides enough space for the coupling apertures.

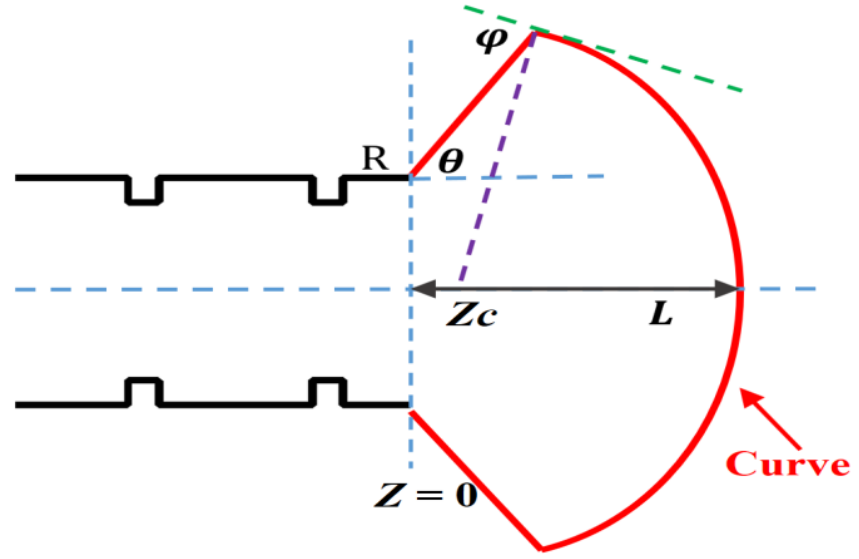

Fig. 3. Definition of the 'zero-field' boundary.

The position $L$ with minimum electric field strength was calculated from the field strength distribution along the $\mathrm{Z}$ axis. Since its interval inside the corrugated waveguide is half of the waveguide wavelength $\lambda_{\mathrm{g}} / 2$ and its interval is half of the freespace wavelength $\lambda / 2$ in the far-field, it is reasonable to rewrite $\mathrm{L}$ as the function of the number of half periods $N$ and to provide universal and scalable results, as shown in Eq. 2.

$$
\mathrm{L}(N)=\frac{\lambda}{2} N\left[1+\mathrm{F}(N) \frac{\lambda \mathrm{g}}{\lambda}\right]
$$

The corrugated waveguide is normally overmoded. Therefore $\lambda_{\mathrm{g}}$ and $\lambda$ have similar magnitudes. In this study for the $36 \mathrm{GHz}$ corrugated waveguide, $\lambda_{\mathrm{g}}=9.06 \mathrm{~mm}$ and $\lambda=$ $8.33 \mathrm{~mm}$ [12]. $\mathrm{F}(N)$ is a correction function, whose value is small and can be obtained numerically by processing the FDTD simulation results. It is larger at the first few half periods and then becomes smaller. The fitted function plotted in Fig. 4 can be written as in Eq. 3.

$$
\mathrm{F}(N)=0.123 \mathrm{e}^{-0.213 N}+0.015
$$

In a practical design, $N \geq 6$ can be used to give a small $\mathrm{F}(N)$ value, as well as to provide more space for the coupling apertures. When $N=8$, the contour lines of $-40 \mathrm{~dB}$ are shown in Fig. 5. The taper angle after linear curve fitting was $53^{\circ}$. It denoted the minimum taper angle. A larger taper angle would be better however it only had a small effect on the cavity Q factor and resonance frequency because the field strength was very small. Adjusting the taper angle could be an effective approach for fine tuning the resonance frequency.

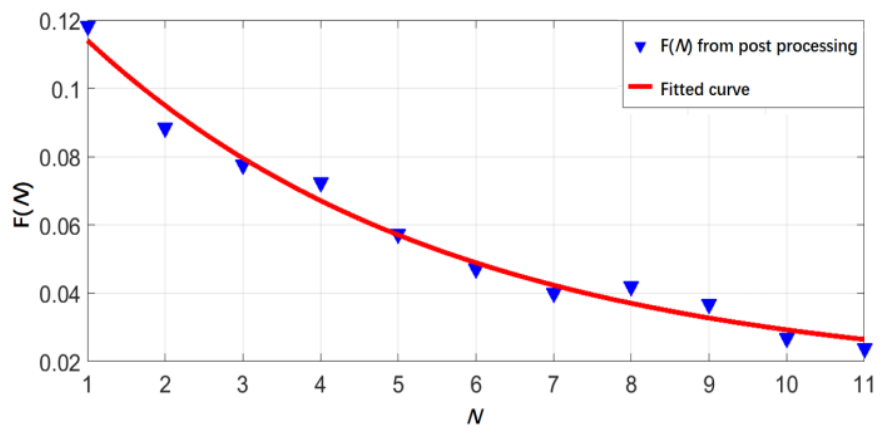

Fig. 4 The values of the correction function $\mathrm{F}(N)$.

The curved profile of the end cap was also studied at different $N$ values by fitting with elliptic and parabolic functions. It was found that the curves matched perfectly with a circle at lower $N$ in the range of 6 to 8 , and a low ellipticity of 0.24 at higher $N$ larger than 8 as shown in Fig. 6 . Further study showed that the angle $\varphi$ was close to $90^{\circ}$, therefore the center of the radius of the circle $\mathrm{Z}_{c}$ could be estimated using

$$
\mathrm{Z}_{c}=-\mathrm{R} / \tan \theta
$$

where $\mathrm{R}$ is the aperture radius of the corrugated waveguide.

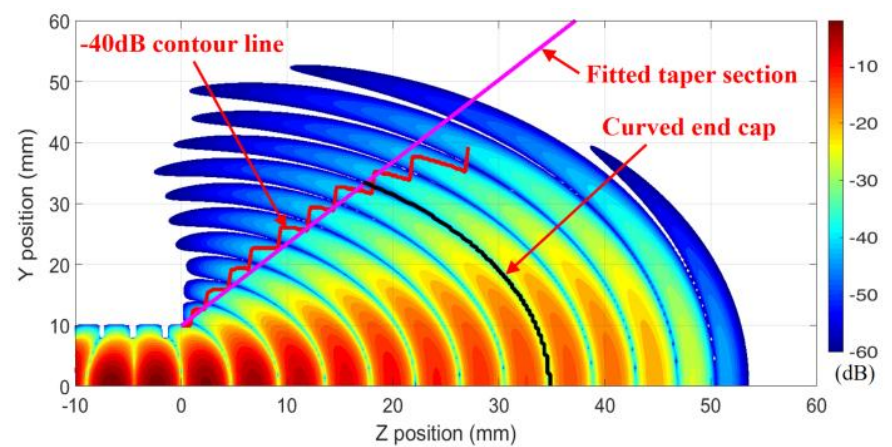

Fig. 5. The taper and end cap structure from the simulation.

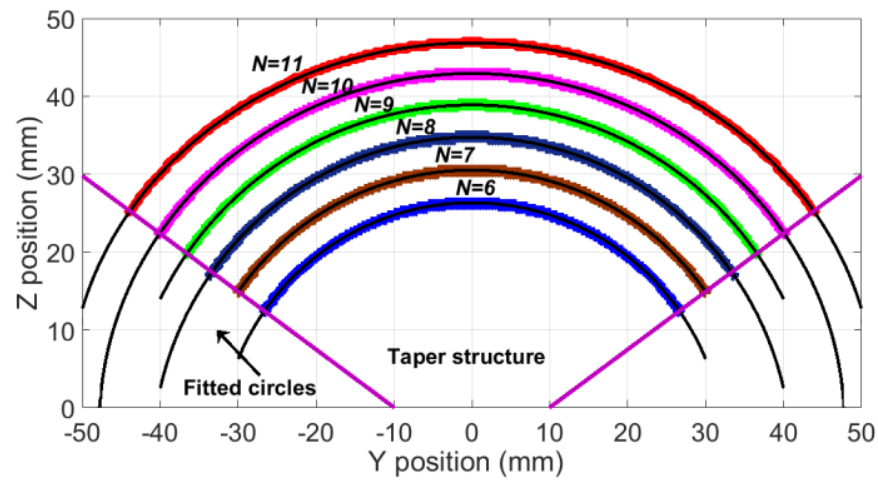

Fig. 6 The shapes of end caps for different $N$ values and the fitted circles.

Besides the electric field pattern studied with the minimum strength at $Z=0$ as shown in Fig. 7(a), another typical field pattern is the maximum field strength at $Z=0$, as shown in Fig. 7(b). This corresponds to an additional length of $\lambda_{\mathrm{g}} / 4$ at both sides of the corrugated waveguide. The same analysis was carried out in this case, however Eqs. 2-4 should be replaced by Eqs. 5-7 with the previous assumptions still valid. Since there 
is no significant difference between these two cases, only the results of the first case are presented in the following sections.

The $\mathrm{L}(N)$ was revised as

$$
\mathrm{L}(N)=\frac{\lambda}{2} N\left[1+\mathrm{F}(N) \frac{\lambda_{\mathrm{g}}}{\lambda}\right]-\frac{\lambda_{\mathrm{g}}}{4}
$$

The correction function $\mathrm{F}(N)$ at different $N$ is shown in Fig. 8 and it only has slight differences compared with Fig. 4. The fitted function is

$$
\mathrm{F}(N)=0.153 \mathrm{e}^{-0.179 N}+0.008
$$

The taper angle is also $53^{\circ}$, and the center of the circle for the end cap becomes

$$
\mathrm{Z}_{c}=-\left(\mathrm{R} / \tan \theta-\lambda_{g} / 4\right)
$$

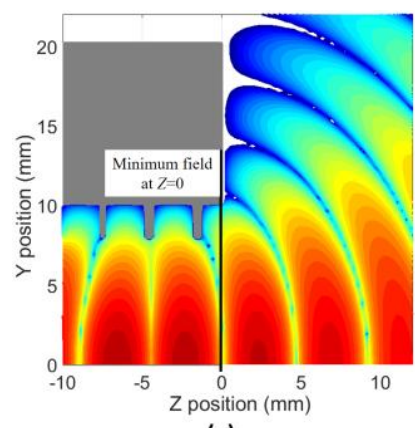

(a)

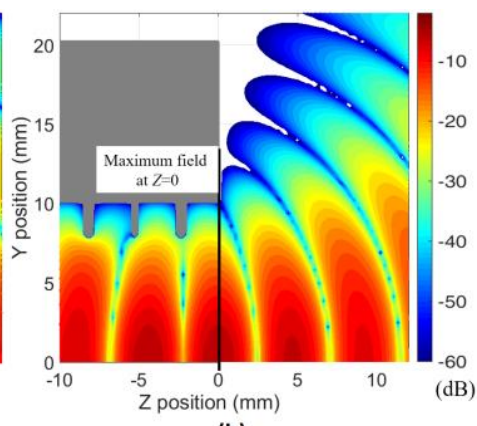

(b)
Fig. 7 The shapes of end caps at different $N$ values and the fitted circles.

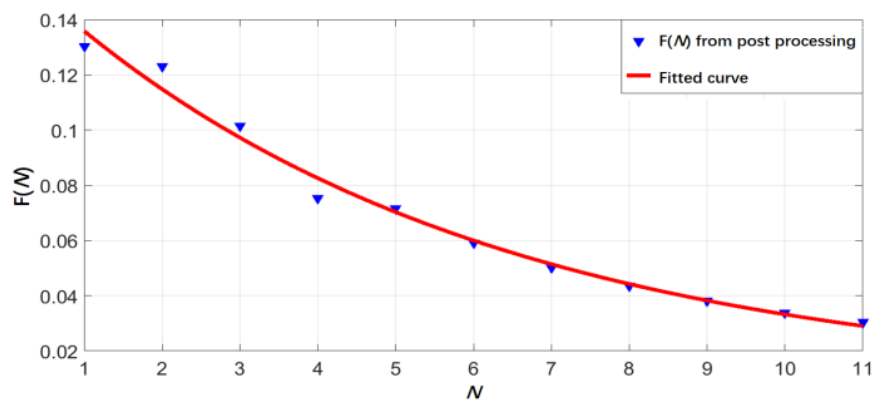

Fig. 8 The fitted function of $\mathrm{F}(N)$.

\section{THE SIMULATION RESULTS OF THE CORRUGATED CAVITY}

With the analysis from the previous sections, the complete microwave undulator cavity was simulated. To save the simulation time, 36 periods of the corrugated waveguide were used in the simulation using CST Microwave Studio. The eigenmode solver was used to calculate the resonance frequencies close to the designed operating frequency of $36 \mathrm{GHz}$. Tetrahedral rather than hexahedral meshes were used to accurately present the curved coupling structure. Oxygenfree high-conductivity copper, with conductivity of $5.8 \times$ $10^{7} \mathrm{Sm}^{-1}$, was chosen as the metal material in the simulation to calculate the $\mathrm{Q}$ factor of the corrugated cavity. $N=6$ was chosen to calculate the other geometry parameters. Parameter sweeps were used to determine the final parameters as well as their sensitivity. The results are shown in Fig. 9.

The most sensitive parameter was $L$. The cavity size increased as it increased, and resulted in the reduction in the eigenfrequency. There was $\sim 0.3 \mathrm{~mm}$ tuning region of $L$, corresponding to a frequency tuning range of $0.13 \mathrm{GHz}$, where the $\mathrm{Q}$ factor did not change significantly. Tuning the position of $\mathrm{Z}_{c}$ had similar performance with $L$ however it was not as sensitive compared with $L$. When $\Delta \mathrm{Z}_{c}=-0.3 \mathrm{~mm}$, the maximum Q factor was achieved. As had been predicted, the eigenfrequency and $\mathrm{Q}$ factor were insensitive to the taper angle as long as its value was larger than $44^{\circ}$. The parameter scan showed that the maximum $\mathrm{Q}$ factor could be achieved when $\theta$ was in the range of $48^{\circ}$ to $52^{\circ}$. At a larger angle, although the eigenfrequency did not change much, the $\mathrm{Q}$ factor dropped quickly because of the field pattern distortion. The final parameter set was chosen from the parameter scan results in the sequence of $\mathrm{L}, \mathrm{Z}_{c}$ and $\theta$. Table 1 shows the comparison of the parameter values and their performance from the empirical equations and final chosen values. In both cases, the most sensitive parameter $L$ were very close in value. The difference in the eigenfrequency was negligible. The improvement on the $\mathrm{Q}$ factor was about $3.4 \%$.

TABLE I Comparison of the initial parameter values and the optimized results

\begin{tabular}{|l|l|l|}
\hline Parameters & Initial values & Optimized values \\
\hline$R$ & $10.10 \mathrm{~mm}$ & $10.10 \mathrm{~mm}$ \\
\hline$L$ & $26.33 \mathrm{~mm}$ & $26.30 \mathrm{~mm}$ \\
\hline$Z c$ & $-7.61 \mathrm{~mm}$ & $-7.91 \mathrm{~mm}$ \\
\hline$\theta$ & $53.0^{\circ}$ & $50.8^{\circ}$ \\
\hline$\varphi$ & $90.0^{\circ}$ & $90.0^{\circ}$ \\
\hline Eigen frequency & 36.01 & 36.02 \\
\hline Q factor & 86210.1 & 89122.5 \\
\hline
\end{tabular}

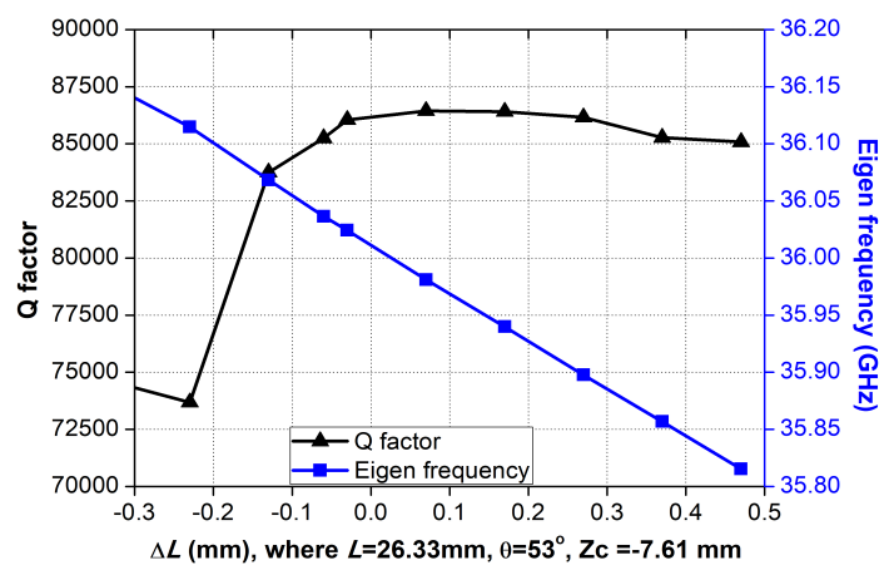

(a)

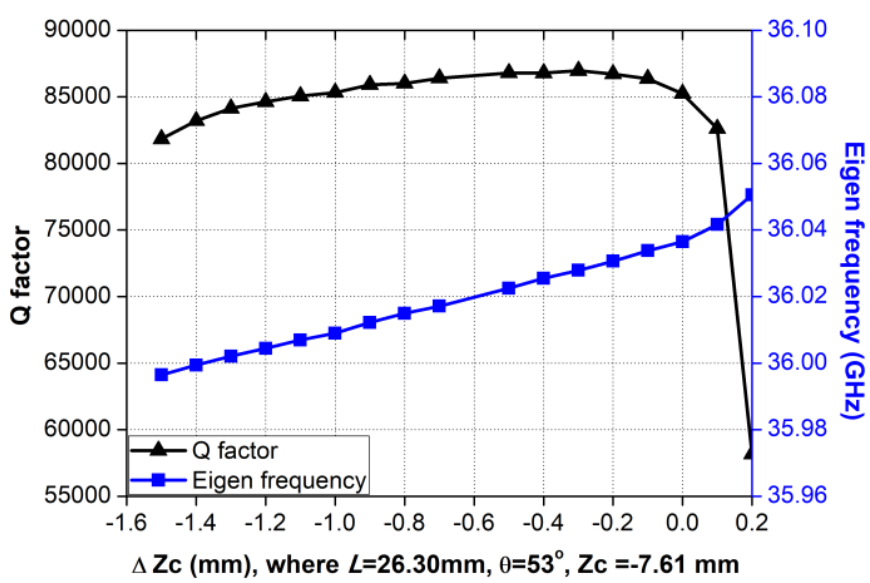

(b) 


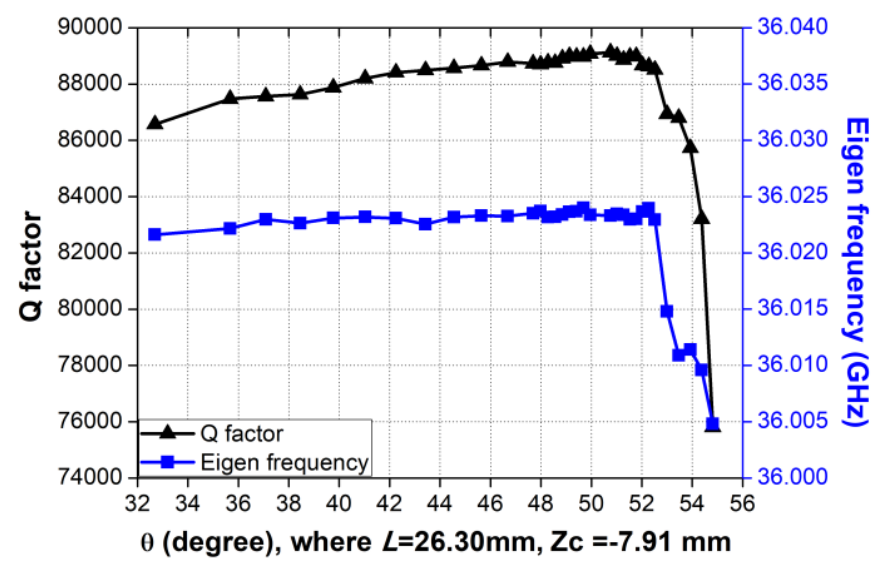

(c)

Fig. 9 Results of sweeping the geometry parameters.

The design had minimized the effect of the end caps on the cavity was well as keeping the resonance frequency unchanged, it showed good scalability with regard to a different number of periods of the corrugated waveguide section, as shown in Fig. 10. The difference in the resonance frequency of less than $0.04 \%$ was observed. Because the end caps have larger Ohmic loss than the corrugated waveguide, when the period number increases, the average loss per unit length decreases, therefore the $\mathrm{Q}$ factor increases. The distribution of the electric field of the microwave undulator cavity with 72 regular periods on the $\mathrm{X}-\mathrm{Z}$ plane is shown in Fig. 11. The coupling apertures of standard WR28 waveguide were placed at the position of minimum electric field, and its impact on the cavity properties can be neglected.

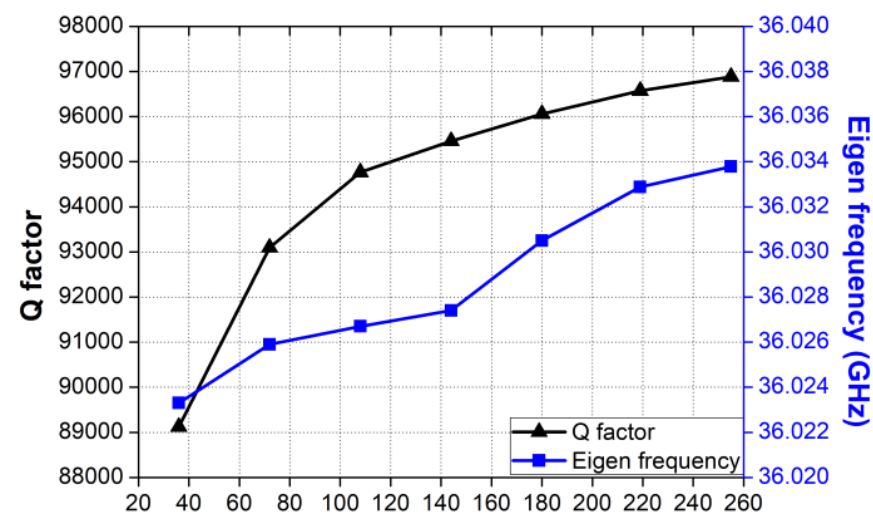

Period number of the corrugated waveguide

Fig. 10 Cavity $\mathrm{Q}$ factors and eigenfrequencies at different period number of the corrugated waveguide.

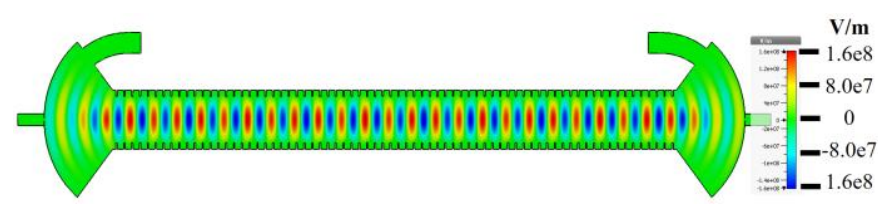

Fig. 11 Electric field pattern of the microwave undulator with 72 regular periods.

\section{CONCLUSION}

In this paper, a set of empirical equations was obtained from the FDTD simulation to get a fast prediction of the geometric parameters of the microwave undulator using a corrugated waveguide. The equations were normalized to the free-space wavelength therefore it is scalable for different operating frequencies. The design of the end caps and coupling aperture has minimum effect on the cavity resonance frequency and quality factor. Therefore, it is also scalable for different period numbers.

The same design process can be used for a cavity operating with higher order modes to increase the dimensional size when the microwave undulator operates at a higher frequency. For example, the $\mathrm{HE}_{12}$ mode has a waveguide radius 2.5 times larger than when operating with a $\mathrm{HE}_{11}$ mode, but this will significantly increase the simulation time. The method presented in this paper can help to shorten the time used to design the microwave undulator cavity.

\section{REFERENCES}

D. A. G. Deacon, L. R. Elias, J. M. J. Madey, G. J. Ramian, H. A. Schwettman, and T. I. Smith, "First Operation of a Free-Electron Laser," Phys. Rev. Lett., vol. 38, no. 16, pp. 892-894, April 1977. DOI: 10.1103/PhysRevLett.38.892 theory," Physical Review Special Topics - Accelerators and Beams, vol. 10, no. 3, p. 034801, March 2007. DOI: 10.1103/PhysRevSTAB.10.034801

T. Shintake, K. Huke, J. Tanaka, I. Sato, and I. Kumabe, "Microwave Undulator," Jpn. J. Appl. Phys., vol. 21, no. 10A, pp. L601-L603, 1982. DOI: 10.1143/JJAP.21.L601

T. Shintake, K. Huke, J. Tanaka, I. Sato, and I. Kumabe, "Development of Microwave Undulator," Jpn. J. Appl. Phys., vol. 22, no. 5R, pp. 844-851, May 1983. DOI: 10.1143/JJAP.22.844

J. A. Clarke, The Science and Technology of Undulators and Wigglers. Oxford University Press, 2004.

S. O. Prestemon, D. R. Dietderich, S. E. Bartlett, M. Coleman, S. A. Gourlay, A. F. Lietzke, S. Marks, S. Mattafirri, R. M. Scanlan, R. D. Schlueter, B. Wahrer, and B. Wang, "Design, fabrication, and test results of undulators using $\mathrm{Nb} / \mathrm{sub} 3 / \mathrm{Sn}$ superconductor," IEEE Transactions on Applied Superconductivity, vol. 15, no. 2, pp. 12361239, 2005. DOI: 10.1109/TASC.2005.849540

M. Seidel, "Parameter evaluation for microwave undulator schemes," DESY-TESLA-FEL-2001-08, 2001.

S. V. Kuzikov, Y. Jiang, T. C. Marshall, G. V. Sotnikov, and J. L. Hirshfield, "Configurations for short period rf undulators," Physical Review Special Topics - Accelerators and Beams, vol. 16, no. 7, p. 070701, 07/19/ 2013. DOI: 10.1103/PhysRevSTAB.16.070701

S. V. Kuzikov, A. V. Savilov, and A. A. Vikharev, "Flying radio frequency undulator," Appl. Phys. Lett., vol. 105, no. 3, p. 033504, 2014/07/21 2014. DOI: $10.1063 / 1.4890586$

M. Shumail, "Theory, design, and demonstration of a new microwave-based undulator," $\mathrm{PhD}$, Department of Electrical Engineering, Stanford University, 2014.

S. Tantawi, M. Shumail, J. Neilson, G. Bowden, C. Chang, E. Hemsing, and M. Dunning, "Experimental Demonstration of a Tunable Microwave Undulator," Phys. Rev. Lett., vol. 112, no. 16, p. 164802, April 2014. DOI: 10.1103/PhysRevLett.112.164802 L. Zhang, W. He, J. Clarke, K. Ronald, A. D. R. Phelps, and A. Cross, "Systematic study of a corrugated waveguide as a microwave undulator," Journal of Synchrotron Radiation, vol. 26, no. 1, 2019. DOI: doi:10.1107/S1600577518014297

F. Toufexis and S. Tantawi, "A $1.75 \mathrm{~mm}$ Period RF-Driven Undulator," in Proceedings, 8th International Particle Accelerator Conference (IPAC 2017), Copenhagen, Denmark, 2017, pp. TUPAB135-TUPAB135, 2017.

F. Toufexis, J. Neilson, and S. Tantawi, "Coupling and Polarization Control in a mm-wave Undulator," in Proceedings, 8th International Particle Accelerator Conference (IPAC 2017): Copenhagen, Denmark, May 14-19, 2017, 2017, pp. TUPAB136TUPAB136, 2017.

V. Paul Scherrer Institute, "SwissFEL - Conceptual design report," Switzerland2010. 
[16] E. J. Kowalski, D. S. Tax, M. A. Shapiro, J. R. Sirigiri, R. J. Temkin, T. S. Bigelow, and D. A. Rasmussen, "Linearly Polarized Modes of a Corrugated Metallic Waveguide," IEEE Trans. Microwave Theory Techn., vol. 58, no. 11, pp. 2772-2780, 2010. DOI: 10.1109/TMTT.2010.2078972

[17] P. J. B. Clarricoats and A. D. Olver, Corrugated Horns for Microwave Antennas. Institution of Engineering and Technology, 1984.

[18] G. L. James, "Analysis and Design of TE11-to-HE11 Corrugated Cylindrical Waveguide Mode Converters," IEEE Trans. Microwave Theory Techn., vol. 29, no. 10, pp. 1059-1066, 1981. DOI: 10.1109/TMTT.1981.1130499

[19] J. M. Neilson, P. E. Latham, M. Caplan, and W. G. Lawson, "Determination of the resonant frequencies in a complex cavity using the scattering matrix formulation," IEEE Trans. Microwave Theory Techn., vol. 37, no. 8, pp. 1165-1170, 1989. DOI: $10.1109 / 22.31074$

[20] L. Zhang, W. He, C. R. Donaldson, and A. W. Cross, "Bandwidth Study of the Microwave Reflectors with Rectangular Corrugations," Journal of Infrared, Millimeter, and Terahertz Waves, journal article vol. 37, no. 9, pp. 846-856, September 01 2016. DOI: 10.1007/s10762-016-0280-y

[21] P. J. B. Clarricoats and P. K. Saha, "Propagation and radiation behaviour of corrugated feeds. Part 2: Corrugated-conical-horn feed," Electrical Engineers, Proceedings of the Institution of, vol. 118, no. 9, pp. 1177-1186, 1971. DOI: 10.1049/piee.1971.0212

[22] T. Liebig, A. Rennings, S. Held, and D. Erni, "openEMS - a free and open source equivalent-circuit (EC) FDTD simulation platform supporting cylindrical coordinates suitable for the analysis of traveling wave MRI applications," International Journal of Numerical Modelling: Electronic Networks, Devices and Fields, vol. 26, no. 6, pp. 680-696, 2013. DOI: doi:10.1002/jnm.1875

[23] M. Shumail, G. B. Bowden, C. Chang, J. Neilson, S. G. Tantawi, and C. Pellegrini, "Application of the Balanced Hybrid Mode in Overmoded Corrugated Waveguides to Short Wavelength Dynamic Undulators," Conf. Proc., vol. THPC183, pp. 3326-3328, 2011. 